\title{
A Multi Item Inventory Model for Deteriorating Items with Expiration Date and Allowable Shortages
}

\author{
Shilpy Tayal ${ }^{*}$, S. R. Singh ${ }^{2}$ and Rajendra Sharma ${ }^{1}$ \\ 'Department of Mathematics, Graphic Era University, Dehradun, India; \\ agarwal_shilpy83@yahoo.com,rs.2103@yahoo.in \\ 2Department of Mathematics, DN College, Meerut, India; shivrajpundir@gmail.com
}

\begin{abstract}
Inventory decisions in supply chain are crucial for its success. These decisions become more important for the products with expiration date. Making these decisions in inventory systems with multiple products is a challenging task for managers. Most approaches in the literature for optimizing decisions in such an environment consider only a single item inventory. This paper presents a multi item inventory model to optimize the unit time profit of inventory management for the products having an expiration date after which the product can not be sold. As on one side the shortage costs are significant, on the other side, to maintain appropriate inventory levels for such type of products and avoid shortages is a very problematic job. For validation, the model is simulated and the results are compared. This article offers an approach for optimization and thus has business significance.
\end{abstract}

Keywords: Deterioration, Expiration Date, Lead Time, Multi Items, Partial Backlogging, Shortages

\section{Introduction}

Supply chain for the products with high fluctuation in demand patterns and a fix time of useable period necessitates better and at times different planning approach for market, based on forecast data. The shortage costs in such cases are significant and any loss in sales badly impacts on the balance sheet. The problem gets compounded when the executives are required to deal with variety. The model presents a new scheme to arrive at the inventory replenishment levels and tries to improve the pull in the system. This is usually taken care of by maintaining high inventory levels at the cost. It may become even more complicated if the deterioration rates and lead time for the items are also taken into account. For such requirements, the conventional optimization models based on inventory costs are extremely difficult to practice. The modification in the periodic review with the cyclic replenishments based on forecasts and the rhythm followed may offer a better way of modeling the inventory patterns in system to take care of real-life issues like shortage. The behaviour of the demand and the forecasting method employed provides an approach to obtain the optimal ordering quantity. The ultimate aim of inventory management is to serve the customers with highest possible service levels and flexibility keeping the nature of the system in consideration. Many of the classical inventory models concern with single-item model. In fact such this model seldom occurs. Ben-Daya and Raouf ${ }^{1}$ have developed approach for a more realistic and general SPIP (Single Period Inventory Problem), they consider a multi item with budgetary and floor- or shelf- space constraints, they assume that, the demand of the items follows uniform probability distribution. Also, they have discussed a multi-item inventory model with stochastic demand subject to the restrictions on available space and budget. Bhattacharya ${ }^{2}$ has studied a two item inventory for deteriorating items with a linear stock-dependent demand

*Author for correspondence 
rates. Lenard and $\operatorname{Roy}^{3}$ are define another approach for the determination of inventory policies based on the notion of efficient policy surface and extend this notion to multiitem inventory control by defining the concepts of family and aggregate item. Mathematicians like Worell and Hall ${ }^{4}$ have applied different programming methods to solve multi-items inventory problems. Sulem ${ }^{5}$ has been determined the optimal ordering policy for impulse control of a deterministic two product inventory system subject to constant demand rates, linear storage and shortage costs and economies of joint ordering.

An important assumption in inventory models found in the existing literature is that the lifetime of an item is infinite while it is in storage. But the effect of deterioration plays an important role in the storage of some commonly used decaying items like, breakable items, (glass, china clay, ceramic goods etc.), radioactive substances, perishable goods etc. In these cases, a certain fraction of these goods are either damaged or decayed and are not in a perfect condition to satisfy the future demand of customers for good items. Deterioration in such items is continuous and time independent or time dependent and/or dependent on on-hand inventory. A number of research papers have already been published on above type of items.

Goswami and Chaudhuri ${ }^{6}$ put forward a model with and without shortages by assuming a linear time dependent demand. Bhunia and Maiti ${ }^{7}$ corrected this model for its shortcomings and considered deterioration in the stock. Singh and Singh ${ }^{8}$ developed a continuous production control inventory model for deteriorating items with linear demand rate in the environment of permissible delay of payments. Tayal et al. ${ }^{9}$ developed a two echelon supply chain model for deteriorating items with effective investment in preservation technology. Singh and Singh ${ }^{10}$ assumed optimal ordering policy for decaying items with stock-dependent demand under inflation in a supply chain. Singh and Jain ${ }^{11}$ explored a deterministic inventory model for a deteriorating item in an inflation-induced environment. Hsu et al. ${ }^{12}$ presented an optimal ordering decision for deteriorating items with expiration date and uncertain lead time in which the demand for the product decreases as the product is nearer to the expiration date.

Now we discuss another feature which comes frequently in realistic business environment which is shortage. It plays an important role and creates backlogging. There are two type of backlogging; (a) complete backlogging, (b) partial backlogging. In the most of the referred papers, complete backlogging of unsatisfied demand is assumed. In practice, there are customers who are willing to wait and receive their orders at the end of shortage period, while other is not. Mandal and $\mathrm{Pal}^{13}$ considered inventory model for exponentially decaying items by allowed shortages. Wu et al. ${ }^{14}$ related the backlogging rate to the waiting time up to the next replenishment. In the last few years, considerable attention has been paid to inventory models with partial as well as complete backlogging. The backlogging rate can be modeled taking into account the customer's behavior. The first paper in which customer's importance functions are proposed seems to be that by $\mathrm{Abad}^{15}$. Change and Dye ${ }^{16}$ developed a finite horizon inventory model using Abad's reciprocal backlogging rate. Singh et al. ${ }^{17}$ presented an EOQ model for perishable items with power demand and partial backlogging. Arya et al. ${ }^{18}$ developed an order level inventory model for perishable items with stock dependent demand and partial backlogging. Here in this work we have used partial backlogging which depends on waiting time. The demand is taken as a function of price and expiration date. Singh and Vishnoi ${ }^{19}$ introduced a supply chain inventory model for deteriorating and ameliorating items with price-dependent consumption rate. Singh et al. ${ }^{20}$ a soft computing based inventory model with deterioration and price dependent demand.

In this paper consideration was given to the control of multiproduct inventory under deterministic demands. The multi-item inventory models are more realistic than the single item model. So this study concern with three item inventory models. The purpose remains the same for single-item as well as for multi-item inventory. The analysis for a single-item inventory is almost parallel to that of multi-item inventory. This article considers the real-life requirements like product variety with expiration date and provides a simple as well as logical method which may be used for the inventory optimization to arrive at the overall results without tedious calculations. Much attention has been devoted to variant inventory models, but no more models with expiration date for multi items are found in the literature. Since in real life there are so many products which have a fix life cycle, and after which they cannot be used. Demand for these product decreases as the product is nearer to expiration date. To maintain appropriate inventory levels for such type of products and avoid shortages is a very problematic job. This model is treated with multiple items having a fix expiration date 
for each product. The proposed model takes care of real-life business requirements. The empirical relation in the proposed model is used to calculate the items variability with a set of assumptions that are practically acceptable. There is no simple model that offers the optimal result for the variable quantity with expiration date.

In the next section we present the multi item inventory model and derive the optimal control of the system. The present paper is organized as follow. Section 2 includes the assumptions and notations of this model. Section 3 is devoted to obtain the mathematical formulation of the optimal control problem of this model. In section 4 optimality of the model is proved. Section 5 provides a numerical example to clarify the proposed model. Sensitivity analysis is presented in section 6. Finally observations and conclusion of the results are presented.

\section{Assumptions and Notations}

We consider a multi item inventory model with the following notation and assumptions for the products having a fix expiration date after that which can not be used.

1. Deterioration rate for the product is assumed as $\theta_{i} t_{i}$ which is a linear function of time.

2. Demand rate for the product follows a function of price $\mathrm{p}_{\mathrm{i}}$ and expiration date $\mathrm{T}_{\mathrm{i}}$ and is given by $\mathrm{D}_{\mathrm{i}}(\mathrm{t})=$ $\frac{\left(T_{i}-t\right)}{\left(p_{i}^{\beta}-1\right)}$, where $0<\beta<1$,

3. The model is considered for multi items.

4. Shortages are allowed and partially backlogged at the rate of $\mathrm{B}(\eta)$ where $B(\eta)=K_{i 0} e^{-K_{i 1} \eta}, \mathrm{K}_{\mathrm{i} 0}<1$ and $\mathrm{K}_{\mathrm{i} 1} \geq 0$.

5. The warehouse has unlimited capacity.

6. There is no replacement or repair of deteriorated items during a given cycle.

7. The shortage period can not exceed the cycle time.

$\mathrm{T}_{\mathrm{i}} \quad$ expiration date for the ith product

$\mathrm{y}_{\mathrm{i}} \quad$ lead time for the ith product

$\mathrm{v}_{\mathrm{i}} \quad$ time at which inventory level becomes zero

$\mathrm{p}_{\mathrm{i}} \quad$ the selling price per unit for the retailer

$c_{i}$ the selling price per unit for the vendor

$c_{i m}$ the manufacturing cost per unit for the vendor

$d_{i} \quad$ deterioration cost per unit for the retailer

$r_{i} \quad$ the lost sale cost per unit for the retailer

$h_{i} \quad$ holding cost per unit per unit time

$\lambda$ a constant
$\mathrm{K}_{\mathrm{i} 0}, \mathrm{~K}_{\mathrm{i} 1}$ positive constants

$\eta \quad$ waiting time upto the next replenishment

$\mathrm{c}_{\mathrm{io}} \quad$ ordering cost for the retailer for ith item

$\theta_{i} \quad$ positive constant

$\xi_{i} \quad$ the vendor's managing cost for ith item

$\mathrm{F}_{\mathrm{iR}} \quad$ unit time profit for ith item for the retailer

$\mathrm{F}_{\mathrm{is}} \quad$ unit time profit for ith item for the vendor

\section{Modelling and Analysis}

\section{Retailer's Model}

\subsection{Case 1}

When the supplier's lead time $y_{i} \leq 0$ :

In this case the supplier completes the order before $y_{i}$ unit of times. Shortages are occurring in the time interval $\left[v_{\mathrm{i}}, T i\right]$. As shown in Figure 1, we consider the following time intervals separately, $[0, v i]$ and $[v i, T i]$. During the interval $[0, v i]$ the inventory level decreases due to combined effect of demand and deterioration. Hence the inventory level for ith item $(0 \leq i \leq k)$ is governed by the following differential equations:

$\begin{array}{ll}\frac{d I_{i 1}(t)}{d t}=-\theta_{i} t I_{i 1}(t)-\frac{\left(T_{i}-t\right)}{\left(p_{i}^{\beta}-1\right)} & 0 \leq t \leq v_{i} \\ \frac{d I_{i 2}(t)}{d t}=-\frac{\left(T_{i}-t\right)}{\left(p_{i}^{\beta}-1\right)} & v_{i} \leq t \leq T_{i}\end{array}$

Using boundary conditions $\mathrm{I}_{\mathrm{i} 1}\left(\mathrm{v}_{\mathrm{i}}\right)=0$ and $\mathrm{I}_{\mathrm{i} 2}\left(\mathrm{v}_{\mathrm{i}}\right)=0$, one can get:

$$
\begin{aligned}
I_{i 1}(t)= & \frac{1}{\left(p_{i}^{\beta}-1\right)}\left\{T_{i}\left(v_{i}-t\right)-\frac{1}{2}\left(v_{i}^{2}-t^{2}\right)+\frac{\theta_{i}}{2}\left(\frac{T_{i}}{3}\left(v_{i}^{3}-t^{3}\right)\right.\right. \\
& \left.\left.-\frac{1}{8}\left(v_{i}^{4}-t^{4}\right)\right)\right\} e^{-\frac{\theta t^{2}}{2}} \quad 0 \leq t \leq v_{i}
\end{aligned}
$$

\section{Inventory}

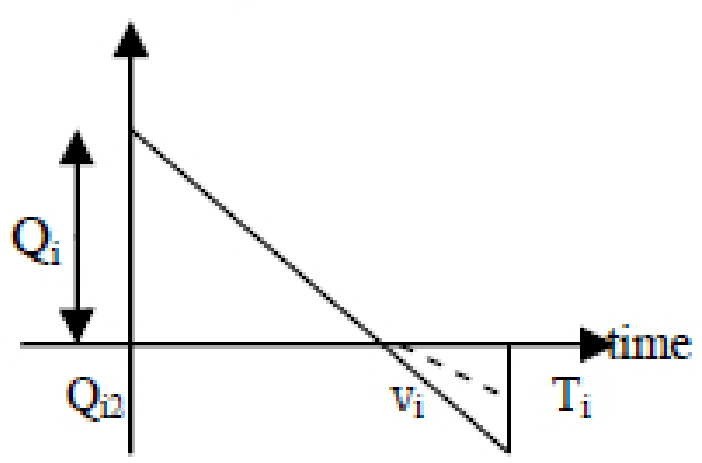

Figure 1. Retailer' inventory level. 
$I_{i 2}(t)=\frac{T_{i}}{\left(p_{i}^{\beta}-1\right)}\left(v_{i}-t\right)-\frac{1}{2\left(p_{i}^{\beta}-1\right)}\left(v_{i}^{2}-t^{2}\right) v_{i} \leq t \leq T_{i}$

From equation (3) using the condition $\mathrm{I}_{1 \mathrm{i}}(0)=\mathrm{Q}_{\mathrm{i}}$ we get:

$Q_{i}=\frac{1}{\left(p_{i}^{\beta}-1\right)}\left\{T_{i} v_{i}-\frac{v_{i}^{2}}{2}+\frac{\theta_{i}}{2}\left(\frac{T_{i} v_{i}^{3}}{3}-\frac{v_{i}^{4}}{8}\right)\right\}$

\section{Vendor's Inventory Model:}

Now let $I_{\text {is }}(t)$ be the supplier's inventory level at $t$ before the beginning of a cycle when the supplier completes the order before $\mathrm{y}_{\mathrm{i}}$ units of time ie $\left(y_{i} \leq 0\right)$ (Figure 2).

The differential equation for the supplier is given by:

$\frac{d I_{i s}(t)}{d t}=-\theta t I_{i s}(t)$

$y_{i} \leq t \leq 0$

Using boundary condition $\mathrm{I}_{\mathrm{is}}(0)=\mathrm{I}_{\mathrm{i} 1}(0)=\mathrm{Q}_{\mathrm{i}}$, one can get:

$I_{i s}(t)=I_{i 1}(0) e^{-\frac{\partial t^{2}}{2}} \quad y_{i} \leq t \leq 0$

When the supplier's delivery is completed early, the retailer's unit time profit for ith item without late delivery is:

$\mathrm{F}_{\mathrm{iR}}=\frac{1}{T}$ [sales revenue - purchasing cost -

$T$ deterioration cost - inventory holding cost - lost sale cost - ordering cost]

Since the supplier suffers inventory holding cost until the target date due to early delivery by $y_{i}$ unit of time, the supplier's unit time profit:

$\mathrm{F}_{\text {is }}(y \leq 0)=\frac{1}{T}$ [sales revenue - purchasing cost cost - managing cost]

$$
\begin{aligned}
F_{i s}(y \leq 0)= & \frac{1}{T}\left[\left(I_{i 1}(0)+Q_{i 2}\right)\left(c_{i}-c_{i m}\right)-\left(I_{i s}\left(y_{i}\right)-I_{i 1}(0)\right) c_{i m}\right. \\
& \left.-h_{i} \int_{y_{i}}^{0} I_{i s}(t) d t-\xi_{i}\right]
\end{aligned}
$$

Sales Revenue $=p_{i} Q_{i 1}+\lambda p_{i} Q_{i 2}$

$$
\begin{aligned}
Q_{i 1} & =\int_{0}^{v_{i}} \frac{\left(T_{i}-t\right)}{\left(p_{i}^{\beta}-1\right)} d t \\
Q_{i 1} & =\frac{v_{i}\left(2 T_{i}-v_{i}\right)}{2\left(p_{i}^{\beta}-1\right)}
\end{aligned}
$$

After the stockout, the arrival of inventory will be of fresh stock, so considering this assumption all the demand during stockout will be of fresh product.

\section{Inventory}

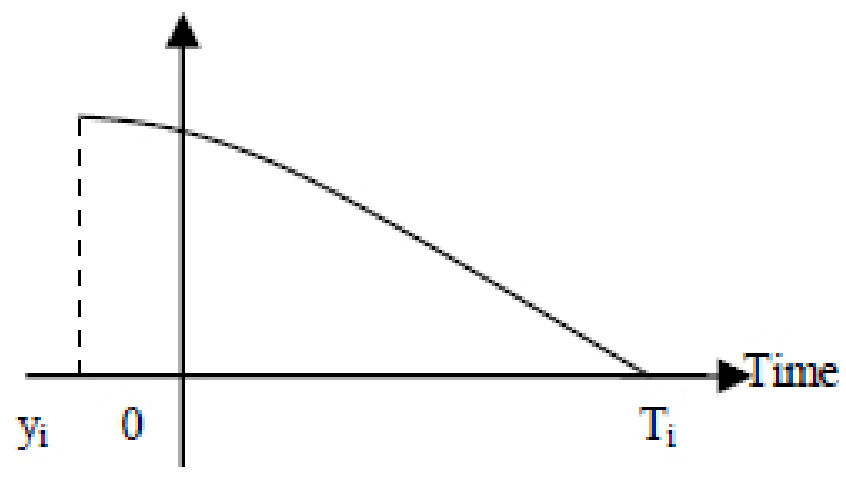

Figure 2. Vendor's inventory level when $y_{i}<=0$.

$$
\begin{aligned}
Q_{i 2} & =\int_{v_{i}}^{T_{i}} \frac{T_{i}}{\left(p_{i}^{\beta}-1\right)} K_{i 0} e^{-K_{i 1}\left(T_{i}-t\right)} \\
Q_{i 2} & =\frac{K_{i 0}}{K_{i 1}} \frac{T_{i}}{\left(p_{i}^{\beta}-1\right)}\left(1-e^{-K_{i 1}\left(T_{i}-v_{i}\right)}\right)
\end{aligned}
$$

The ordering quantity at each replenishment is:

$$
\mathrm{Q}_{\mathrm{i}}=\mathrm{I}_{\mathrm{i} 0}(0)+\mathrm{Q}_{\mathrm{i} 2}
$$

Purchasing cost

$$
\begin{aligned}
& \mathrm{C}_{\mathrm{i}}=\left(\mathrm{I}_{\mathrm{i} 1}(0)+\mathrm{Q}_{\mathrm{i} 2}\right) \cdot c_{\mathrm{i}} \\
& C_{i}= {\left[\frac{1}{\left(p_{i}^{\beta}-1\right)}\left\{T_{i} v_{i}-\frac{1}{2} v_{i}^{2}+\frac{\theta_{i}}{2}\left(\frac{T_{i}}{3} v_{i}^{3}-\frac{v_{i}^{4}}{8}\right)\right\}+\frac{K_{i 0}}{K_{i 1}} \frac{T_{i}}{\left(p_{i}^{\beta}-1\right)}\right.} \\
&\left.\left(1-e^{-K_{i 1}\left(T_{i}-v_{i}\right)}\right)\right] c_{i}
\end{aligned}
$$

The lost sale amount is $=\int_{v_{i}}^{T_{i}} \frac{T_{i}}{\left(p_{i}^{\beta}-1\right)}\left(1-K_{i 0} e^{-K_{i 1}(T-t)}\right) d t$

$$
\text { L.S.C. }=\frac{T_{i}}{\left(p_{i}^{\beta}-1\right)}\left\{T_{i}-v_{i}-\frac{K_{i 0}}{K_{i 1}}\left(1-e^{-K_{i 1}\left(T_{i}-v_{i}\right)}\right)\right\} r_{i}
$$

Deterioration cost $=\left\{I_{i 1}(0)-\int_{0}^{v_{i}} \frac{T_{i}-t}{\left(p_{i}^{\beta}-1\right)} d t\right\} d_{i}$

$$
\text { D.C. }=\frac{1}{\left(p_{i}^{\beta}-1\right)} \frac{\theta_{i}}{2}\left(\frac{T_{i} v_{i}^{3}}{3}-\frac{v_{i}^{4}}{8}\right) d_{i}
$$

Holding cost $=h_{i} \int_{0}^{v_{i}} I_{i 1}(t) d t$

$$
\text { H.C. }=\frac{h_{i}}{\left(p_{i}^{\beta}-1\right)}\left\{\frac{T_{i} v_{i}^{2}}{2}-\frac{v_{i}^{3}}{3}+\frac{\theta_{i} v_{i}^{4} T_{i}}{12}-\frac{\theta_{i} v_{i}^{5}}{60}\right\}
$$

Ordering cost

$$
\text { O.C. }=c_{\text {io }}
$$




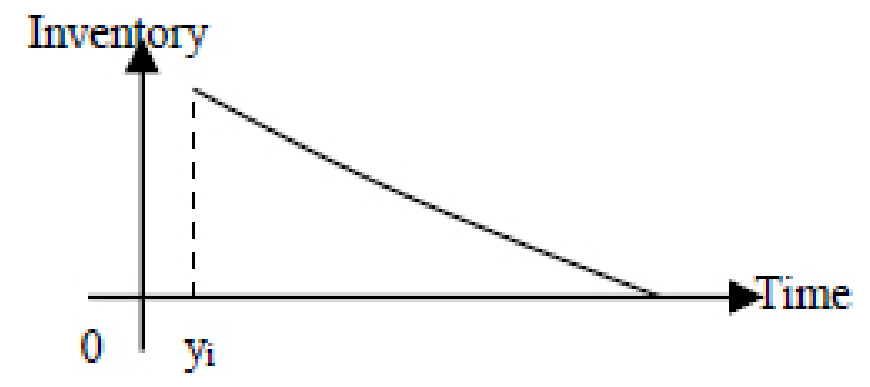

Figure 3. Vendor's inventory level when $y_{i}>0$.

\subsection{Case 2}

When supplier's lead time $y>0$ (Figure 3 ):

The retailer's unit time profit with late delivery by $y$ unit time $\mathrm{F}_{\mathrm{iRd}}$ is:

$F_{i R d}=\frac{1}{T_{i}}$ cost - det cost - holding cost - ordering cost

The retailer maintains his profit regardless of the supplier's delivery behaviour. The supplier's unit time profit for ith item with late delivery by y unit time:

$$
F_{i s d}=\frac{1}{T}\left[\left(I_{i 1}\left(y_{i}\right)+Q_{i 2}\right)\left(c_{i}-c_{i m}\right)-\left(F_{i R}-F_{i R d}\right)-\xi_{i}\right]
$$

sales revenue $=p_{i} Q_{i 1 d}+\lambda p_{i} Q_{i 2}$

$$
\begin{aligned}
Q_{i 1 d} & =\int_{0}^{T_{i}} \frac{\left(T_{i}-t\right)}{\left(p_{i}^{\beta}-1\right)} d t-\int_{0}^{y_{i}} \frac{\left(T_{i}-t\right)}{\left(p_{i}^{\beta}-1\right)} d t \\
Q_{i 1 d} & =\frac{\left(T_{i}-y_{i}\right)^{2}}{2\left(p_{i}^{\beta}-1\right)} \\
Q_{i 2} & =\int_{v_{i}}^{T_{i}} \frac{T_{i}}{\left(p_{i}^{\beta}-1\right)} K_{i 0} e^{-K_{i 1}\left(T_{i}-t\right)} \\
Q_{i 2} & =\frac{K_{i 0}}{K_{i 1}} \frac{T_{i}}{\left(p_{i}^{\beta}-1\right)}\left(1-e^{-K_{i 1}\left(T_{i}-v_{i}\right)}\right)
\end{aligned}
$$

The order quantity at each replenishment is:

$$
\mathrm{Q}_{\mathrm{id}}=\left[\mathrm{I}_{\mathrm{i} 1}\left(\mathrm{y}_{\mathrm{i}}\right)+\mathrm{Q}_{\mathrm{i} 2}\right] \cdot \mathrm{c}_{\mathrm{i}}
$$

The lost sale amount is $=\int_{0}^{y_{i}} \frac{\left(T_{i}-t\right)}{\left(p_{i}^{\beta}-1\right)} d t+\int_{v_{i}}^{T_{i}} \frac{T_{i}}{\left(p_{i}^{\beta}-1\right)}$

The lost sale cost

$$
\left(1-K_{i 0} e^{-K_{i 1}\left(T_{i}-t\right)}\right)
$$

L.S.C. $=\left\{\frac{y_{i}\left(2 T_{i}-y_{i}\right)}{2\left(p_{i}^{\beta}-1\right)}+\frac{T_{i}}{\left(p_{i}^{\beta}-1\right)}\right.$

$$
\left.\left(T_{i}-v_{i}-\frac{K_{i 0}}{K_{i 1}}\left(1-e^{-K_{i 1}\left(T_{i}-v_{i}\right)}\right)\right)\right\} r
$$

Deterioration cost

$$
\begin{aligned}
& \text { D.C. }=\left\{I_{i 1}\left(y_{i}\right)-\int_{y_{i}}^{v_{i}} \frac{\left(T_{i}-t\right)}{\left(p_{i}^{\beta}-1\right)}\right\} d_{i} \\
& \text { D.C. }=\left\{I_{i 1}\left(y_{i}\right)-\frac{2 T_{i}\left(v_{i}-y_{i}\right)-v_{i}^{2}+y_{i}^{2}}{2\left(p_{i}^{\beta}-1\right)}\right\} d_{i}
\end{aligned}
$$

Holding cost

$$
\begin{aligned}
\text { H.C. }= & \int_{y_{i}}^{v_{i}} I_{i 1}(t) d t \\
\text { H.C. }= & \frac{h_{i}}{\left(p_{i}^{\beta}-1\right)}\left[\frac{T_{i} v_{i}^{2}}{2}-\frac{v_{i}^{3}}{3}+\frac{\theta_{i}}{12} T_{i} v_{i}^{4}-\frac{\theta_{i}}{60} v_{i}^{5}\right. \\
& -T_{i}\left(v_{i} y_{i}-\frac{y_{i}^{2}}{2}\right)+\frac{1}{2}\left(v_{i}^{2} y_{i}-\frac{y_{i}^{3}}{3}\right) \\
& -\frac{\theta_{i}}{2}\left\{\frac{T_{i}}{3}\left(v_{i}^{3} y_{i}-\frac{y_{i}^{4}}{4}\right)-\frac{1}{8}\left(v_{i}^{4} y_{i}-\frac{y_{i}^{5}}{5}\right)\right\} \\
& \left.+\frac{\theta_{i} T_{i}}{2}\left(\frac{v_{i} y_{i}^{3}}{3}-\frac{y_{i}^{4}}{4}\right)-\frac{\theta_{i}}{4}\left(\frac{v_{i}^{2} y_{i}^{3}}{3}-\frac{y_{i}^{5}}{5}\right)\right]
\end{aligned}
$$

Ordering cost

O.C $=c_{\text {io }}$

The integrated unit time profit is the sum of unit time profit of vendor and retailer:

$$
\begin{array}{ll}
\mathrm{F}_{\mathrm{i}}=\sum_{i=1}^{k}\left(F_{i R}+F_{i s}\right) & y_{i} \leq 0 \\
\mathrm{~F}_{\mathrm{i}}=\sum_{i=1}^{k}\left(F_{i R d}+F_{i s d}\right) & \mathrm{y}>0
\end{array}
$$

\section{Theorem}

$\mathrm{F}_{\mathrm{i}}$ is concave in $\mathrm{v}_{\mathrm{i}}$ for $h_{i}<\frac{\theta_{i} d_{i} v_{i}}{2}\left(T_{i}-\frac{v_{i}}{2}\right)$ (Figure 4$)$.

Proof:

$$
\begin{aligned}
F_{i}= & p_{i} \frac{v_{i}\left(2 T_{i}-v_{i}\right)}{2\left(p_{i}^{\beta}-1\right)}+\lambda p_{i} \frac{K_{i 0}}{K_{i 1}} \frac{T_{i}}{\left(p_{i}^{\beta}-1\right)}\left(1-e^{-K_{i 1}\left(T_{i}-v_{i}\right)}\right)- \\
& {\left[\frac{1}{\left(p_{i}^{\beta}-1\right)}\left\{T_{i} v_{i}-\frac{1}{2} v_{i}^{2}+\frac{\theta_{i}}{2}\left(\frac{T_{i}}{3} v_{i}^{3}-\frac{v_{i}^{4}}{8}\right)\right\}+\frac{K_{i 0}}{K_{i 1}} \frac{T_{i}}{\left(p_{i}^{\beta}-1\right)}\right.}
\end{aligned}
$$




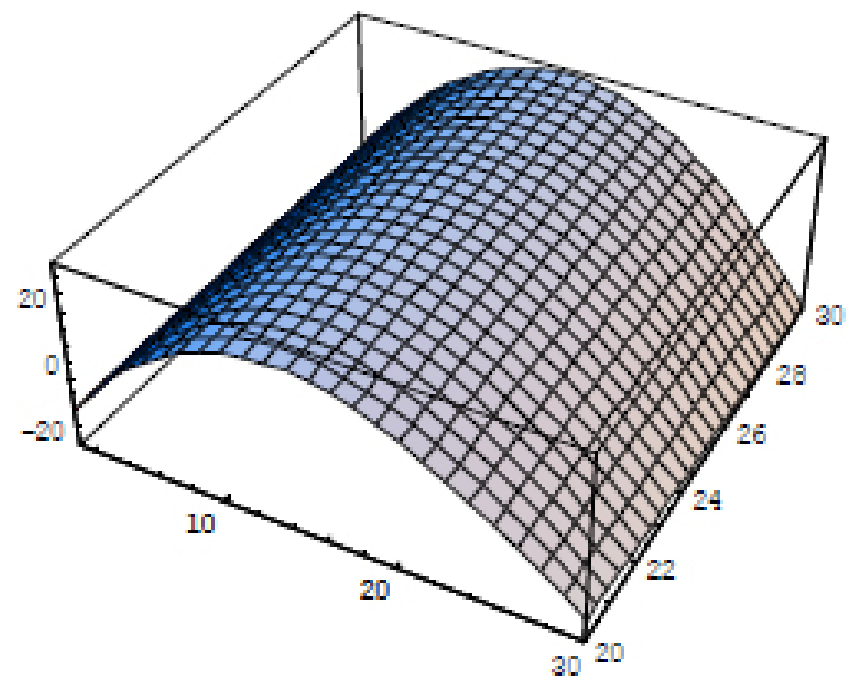

Figure 4. A graphical representation showing the concave function $\mathrm{F}_{\mathrm{i}}(\mathrm{v})$.

$$
\begin{aligned}
& \left.\left(1-e^{-K_{i 1}\left(T_{i}-v_{i}\right)}\right)\right] c_{i}-\frac{T_{i}}{\left(p_{i}^{\beta}-1\right)}\left\{T_{i}-v_{i}-\frac{K_{i 0}}{K_{i 1}}\left(1-e^{-K_{i 1}\left(T_{i}-v_{i}\right)}\right)\right\} r_{i} \\
& -\frac{1}{\left(p_{i}^{\beta}-1\right)} \frac{\theta_{i}}{2}\left(\frac{T_{i} v_{i}^{3}}{3}-\frac{v_{i}^{4}}{8}\right) d_{i}-\frac{h_{i}}{\left(p_{i}^{\beta}-1\right)}\left\{\frac{T_{i} v_{i}^{2}}{2}-\frac{v_{i}^{3}}{3}+\right. \\
& \left.\frac{\theta_{i} v_{i}^{4} T_{i}}{12}-\frac{\theta_{i} v_{i}^{5}}{60}\right\}-\mathrm{c}_{\text {io }} \\
& \frac{\partial F_{i}}{\partial v_{i}}=\frac{p_{i}\left(T_{i}-v_{i}\right)}{\left(p_{i}^{\beta}-1\right)}-\frac{\lambda p_{i} K_{i 0} T_{i}}{\left(p_{i}^{\beta}-1\right)}-\frac{c_{i}}{\left(p_{i}^{\beta}-1\right)}\left\{T_{i}-v_{i}\right. \\
& \left.+\frac{\theta_{i}}{2}\left(T_{i} v_{i}^{2}-\frac{v_{i}^{3}}{2}\right)\right\}-\frac{c_{i} K_{i 0} T_{i}}{\left(p_{i}^{\beta}-1\right)}-\frac{T_{i} r_{i}}{\left(p_{i}^{\beta}-1\right)}\left(K_{i 0}-1\right) \\
& -\frac{\theta_{i} d_{i} v_{i}^{2}}{4\left(p_{i}^{\beta}-1\right)}\left(2 T_{i}-v_{i}\right)-\frac{h_{i}}{\left(p_{i}^{\beta}-1\right)}\left(T_{i} v_{i}-v_{i}^{2}+\frac{\theta_{i} v_{i}^{3} T_{i}}{3}-\frac{\theta_{i} v_{i}^{4}}{12}\right) \\
& \frac{\partial^{2} F_{i}}{\partial v_{i}^{2}}=-\frac{p_{i}}{\left(p_{i}^{\beta}-1\right)}-\frac{c_{i}}{\left(p_{i}^{\beta}-1\right)}\left\{-1+\frac{\theta_{i}}{4}\left(4 T_{i} v_{i}-3 v_{i}^{2}\right)\right\} \\
& -\frac{\theta_{i} d_{i} v_{i}^{2}}{4\left(p_{i}^{\beta}-1\right)}\left(2 T_{i}-v_{i}\right)-\frac{h_{i}}{\left(p_{i}^{\beta}-1\right)}\left(T_{i}-2 v_{i}\right. \\
& \left.+\theta_{i} v_{i}^{2} T_{i}-\frac{\theta_{i} v_{i}^{3}}{3}\right)
\end{aligned}
$$

$$
\begin{aligned}
\frac{\partial^{2} F_{i}}{\partial v_{i}^{2}}= & \frac{1}{\left(p_{i}^{\beta}-1\right)}\left[-p_{i}+c_{i}-\frac{c_{i} \theta_{i} v_{i}}{4}\left(4 T_{i}-3 v_{i}\right)\right] \\
& -\frac{v_{i}}{\left(p_{i}^{\beta}-1\right)}\left\{h_{i}-\frac{\theta_{i} d_{i} v_{i}}{4}\left(2 T_{i}-v_{i}\right)\right\}-\frac{h_{i}}{\left(p_{i}^{\beta}-1\right)}\left\{\left(T_{i}-v_{i}\right)\right. \\
& \left.+\frac{\theta_{i} v_{i}^{3}}{3}\left(T_{i}-\frac{v_{i}^{3}}{3}\right)\right\}
\end{aligned}
$$

\begin{tabular}{|c|c|c|c|}
\hline Item & $1^{\text {st }}$ item & $2^{\text {nd }}$ item & $3^{\text {rd }}$ item \\
\hline $\mathrm{T}_{\mathrm{i}}$ & 20 & 25 & 30 \\
\hline $\mathrm{K}_{\mathrm{i} 0}$ & 0.5 & 0.6 & 0.7 \\
\hline $\mathrm{K}_{\mathrm{i} 1}$ & 1.2 & 1.4 & 1.6 \\
\hline $\mathrm{p}_{\mathrm{i}}$ & 20 & 22 & 24 \\
\hline$\theta_{i}$ & 0.001 & 0.0014 & 0.0018 \\
\hline$\beta$ & 3 & 3 & 3 \\
\hline$y_{i}$ & 2 & 3 & 4 \\
\hline$c_{i}$ & 15 & 16 & 17 \\
\hline$c_{i m}$ & 8 & 9 & 10 \\
\hline $\mathrm{h}_{\mathrm{i}}$ & 0.5 & 0.55 & 0.6 \\
\hline$c_{\text {io }}$ & 200 & 250 & 300 \\
\hline $\mathrm{d}_{\mathrm{i}}$ & 16 & 17 & 18 \\
\hline$r_{i}$ & 8 & 9 & 10 \\
\hline$\xi_{i}$ & 100 & 150 & 200 \\
\hline $\mathrm{k}$ & 3 & 3 & 3 \\
\hline$\lambda$ & 0.8 & 0.8 & 0.8 \\
\hline $\mathrm{v}_{\mathrm{i}}$ & 17.7339 & 15.8353 & 14.558 \\
\hline $\mathrm{I}_{\mathrm{i} 1}(0)$ & 90.6656 & 117.9430 & 137.8010 \\
\hline $\mathrm{Q}_{\mathrm{i} 2}$ & 9.79108 & 46.8878 & 90.2969 \\
\hline $\mathrm{Q}_{\mathrm{i}}$ & 100.4567 & 164.8308 & 228.0979 \\
\hline $\mathrm{Q}_{\mathrm{i} 1}$ & 85.3041 & 110.715 & 128.946 \\
\hline $\mathrm{F}_{\mathrm{iR}}$ & 26.6278 & 27.693 & 32.3593 \\
\hline
\end{tabular}

Here we know that $\mathrm{p}_{\mathrm{i}}>\mathrm{c}_{\mathrm{i}}, \mathrm{T}_{\mathrm{i}}>\mathrm{v}_{\mathrm{i}}$ and for $h_{i}<\frac{\theta_{i} d_{i} v_{i}}{2}\left(T_{i}-\frac{v_{i}}{2}\right)$, $\frac{\partial^{2} F_{i}}{\partial v_{i}^{2}}<0$

This completes the proof.

\section{Numerical Example}

Now we solve the model with the help of partially differentiating the equation with respect to continuous variable $\mathrm{v}_{\mathrm{i}}$ and then obtain the values with the help of numerical using the software mathematica. 


\section{Senstivity Analysis for Variation in Different Parameters}

\begin{tabular}{|c|c|c|c|c|c|c|c|}
\hline Variation & $-15 \%$ & $-10 \%$ & $-5 \%$ & $0 \%$ & $5 \%$ & $10 \%$ & $15 \%$ \\
\hline$\theta 1$ & 0.00085 & 0.0009 & 0.00095 & 0.001 & 0.00105 & 0.0011 & 0.00115 \\
\hline v1 & 18.6459 & 18.3156 & 18.0131 & 17.7339 & 17.4745 & 17.2322 & 17.0048 \\
\hline F1 & 27.864 & 27.433 & 27.0216 & 26.6278 & 26.25 & 25.8867 & 25.5366 \\
\hline$\theta 2$ & 0.00119 & 0.00126 & 0.00133 & 0.0014 & 0.00147 & 0.00154 & 0.00161 \\
\hline v2 & 16.5524 & 16.2986 & 16.0601 & 15.8353 & 15.6228 & 15.4213 & 15.2298 \\
\hline $\mathrm{F} 2$ & 29.0938 & 28.6073 & 28.1409 & 27.693 & 27.2621 & 26.8468 & 26.4461 \\
\hline$\theta 3$ & 0.00153 & 0.00162 & 0.00171 & 0.0018 & 0.00189 & 0.00198 & 0.00207 \\
\hline v3 & 15.2213 & 14.9875 & 14.7668 & 14.558 & 14.3599 & 14.1715 & 13.9919 \\
\hline F3 & 33.9121 & 33.3729 & 32.8559 & 32.3593 & 31.8814 & 31.421 & 30.9768 \\
\hline$\beta 1$ & 0.34 & 0.36 & 0.38 & 0.4 & 0.42 & 0.44 & 0.46 \\
\hline v1 & 16.7355 & 17.0988 & 17.4303 & 17.7339 & 18.0128 & 18.27 & 18.5076 \\
\hline F1 & 37.2107 & 33.174 & 29.6801 & 26.6278 & 23.9398 & 21.5561 & 19.4295 \\
\hline$\beta 2$ & 0.34 & 0.36 & 0.38 & 0.4 & 0.42 & 0.44 & 0.46 \\
\hline v2 & 15.0512 & 15.3427 & 15.6026 & 15.8353 & 16.0447 & 16.2337 & 16.4048 \\
\hline $\mathrm{F} 2$ & 41.8071 & 36.2943 & 31.6485 & 27.693 & 24.2945 & 21.3511 & 18.7834 \\
\hline$\beta 3$ & 0.34 & 0.36 & 0.38 & 0.4 & 0.42 & 0.44 & 0.46 \\
\hline v3 & 13.8235 & 14.0984 & 14.3417 & 14.558 & 14.7513 & 14.9247 & 15.0809 \\
\hline F3 & 51.8192 & 44.0977 & 37.7054 & 32.3593 & 27.8469 & 24.007 & 20.7151 \\
\hline h1 & 0.425 & 0.45 & 0.475 & 0.5 & 0.525 & 0.55 & 0.575 \\
\hline v1 & 18.1615 & 18.0238 & 17.8813 & 17.7339 & 17.5818 & 17.4251 & 17.264 \\
\hline F1 & 28.2725 & 27.7198 & 27.1715 & 26.6278 & 26.0892 & 25.5557 & 25.0277 \\
\hline h2 & 0.4675 & 0.495 & 0.5225 & 0.55 & 0.5775 & 0.605 & 0.6325 \\
\hline v2 & 16.4026 & 16.2116 & 16.0224 & 15.8353 & 15.6503 & 15.4676 & 15.2871 \\
\hline $\mathrm{F} 2$ & 29.2465 & 28.716 & 28.1982 & 27.693 & 27.2002 & 26.7195 & 26.2509 \\
\hline h3 & 0.51 & 0.54 & 0.57 & 0.6 & 0.63 & 0.66 & 0.69 \\
\hline v3 & 15.0697 & 14.8954 & 14.7249 & 14.558 & 14.3947 & 14.2348 & 14.0784 \\
\hline F3 & 33.5004 & 33.1068 & 32.7265 & 32.3593 & 32.0045 & 31.6619 & 31.3311 \\
\hline
\end{tabular}
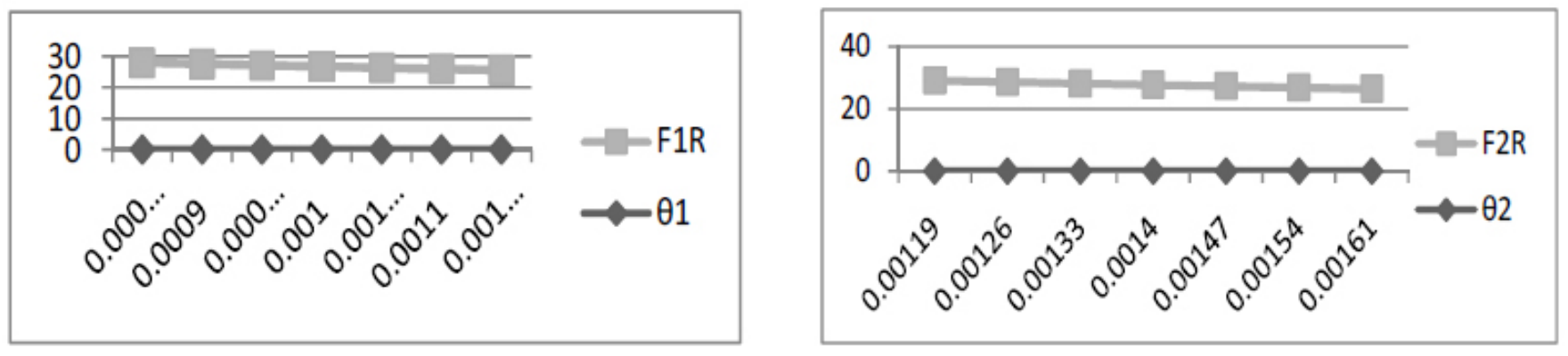

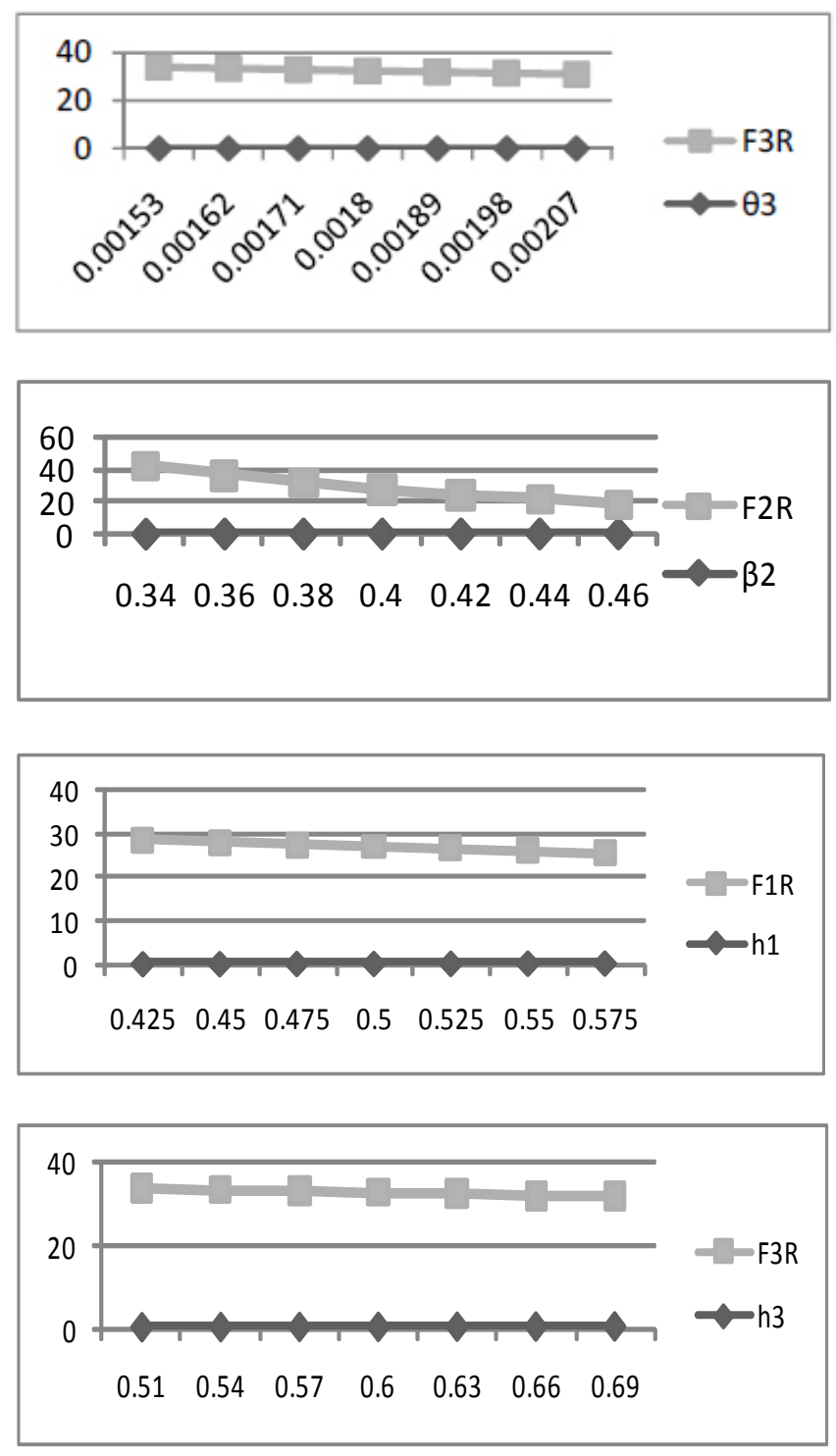

\section{Observations}

From the above tables it can be observed that

1. With the increament in deterioration factor $\theta_{i}$ the unit time profit for all the items decreases.

2. As the value of $\beta_{i}$ increases the unit time profit for all the items decreases.

3. With the increament in holding cost for all the items unit time profit shows the reverse effect.

\section{Conclusion}

The main contribution of this paper has been the development of a dynamic heuristic to determine replenishment cycle and economic order quantity of all the products. The heuristic provides an excellent performance, especially
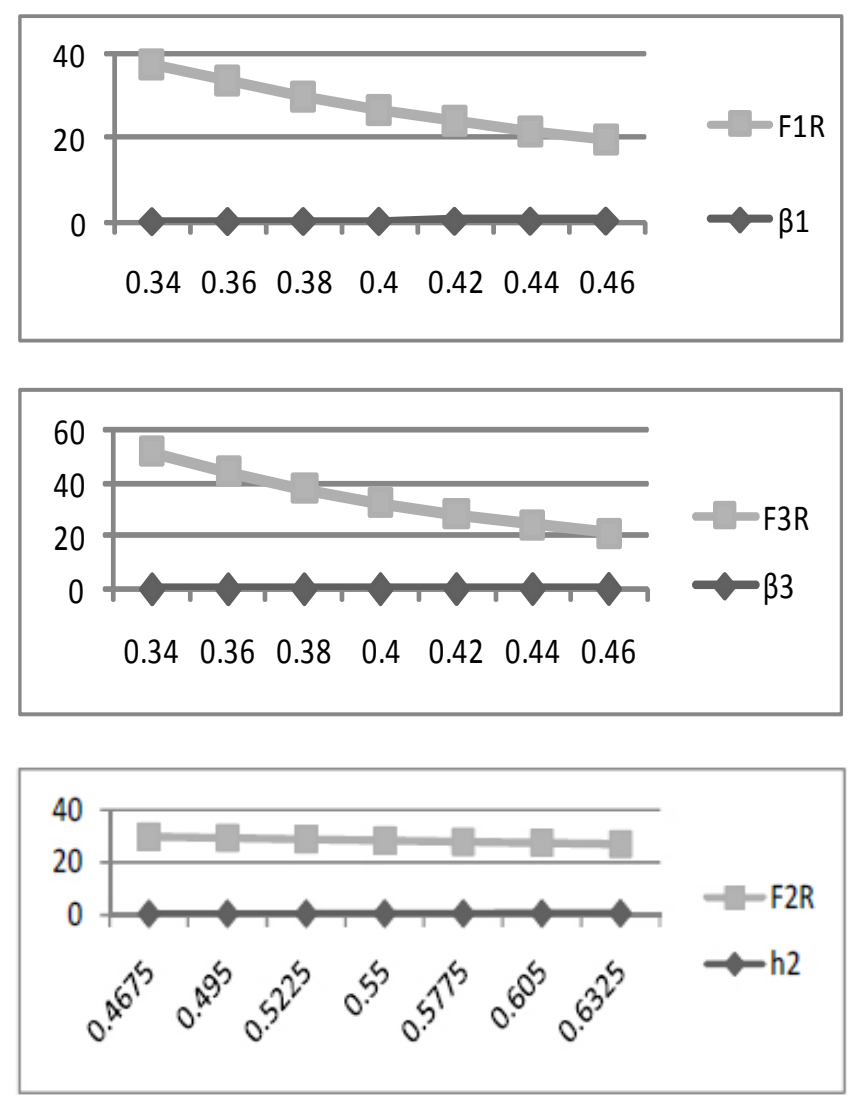

for larger problems which makes it very promising in applications of practical size. A multi-item inventory model of deteriorating items with expiration date is developed and analyzed. As lead time plays very important role in business decision therefore in the present study it is also taken into account. Numerical illustration and sensitivity with respect to different parameters is also presented in the model.

In totality, the setup that has been chosen boasts of uniqueness in terms of the conditions under which the model has been developed. The assumptions of the study impart exclusivity due to the combination of deterioration and expiration date for the products.

The proposed model can be extended in numerous ways. For example, we may extend the demand to a more generalized demand pattern. We could generalize the model to allow for quantity discount. Also, we could consider the unit purchase cost, the inventory holding cost, and others as time dependent.

\section{References}

1. Ben-Daya M, Raouf A. On the constrained multi-item single period inventory problem. International Journal of Production Management. 1993; 13(11):104-112. 
2. Bhattacharya DK. Production, manufacturing and logistics on multi-item inventory. Eur J Oper Res. 2005; 162(3): 786-91.

3. Lenard JD, Roy B. Multi-item inventory control: A multicriteria view. Eur J Oper Res. 1995; 87(3):685-92.

4. Worell EM, Hall MA. The analysis of inventory control model using polynomial geometric programming. International Journal of Production Management. 1982; 20(5):657-67.

5. Sulem A. Explicit solution of a two-dimensional deterministic inventory Problem. Math Oper Res. 1986; 11(1):134-46.

6. Goswami A, Chaudhuri KS. An economic order quantity model for items with two levels of storage for a linear trend in demand. Journal of Operation Research Society. 1992; 43(2):157-67.

7. Bhunia AK, Maiti M. A two warehouse inventory model for a linear trend in demand. Opsearch.1998; 31(4):318-29.

8. Singh SR, Singh N. A production inventory model with variable demand rate for deteriorating items under permissible delay in payments. Int Trans Math Sci Comput. 2009; 2(1):73-82.

9. Tayal S, Singh SR, Sharma R, Chauhan A. Two echelon supply chain model for deteriorating items with effective investment in preservation technology. Int J Mathematics in Operational Research. 2014; 6(1):78-99.

10. Singh SR, Singh C. Optimal ordering policy for decaying items with stock-dependent demand under inflation in a supply chain. International Review of Pure and Advanced Mathematics. 2008; 1(2):31-9.

11. Singh SR, Jain R. Understanding supplier credits in an inflationary environment when reserve money is available. Oper Res. 2009; 6(4):459-74.
12. Hsu PH, Wee HM, Teng HM.Optimal ordering decision for deteriorating items with expiration date and uncertain lead time. Computers \& Industrial Engineering. 2007; 52(4):448-58.

13. Mandel B, Pal AK. Order level inventory system with ramp type demand rate for deteriorating items. J Interdiscipl Math. 1998; 1(1):49-66.

14. Wu JW, Lin C, Tan B, Lee WC. An EOQ inventory model with ramp type demand rate for the items with weibull deterioration. Information and Management Science. 1999; 10(3):41-55.

15. Abad PL. Optimal pricing and lot sizing under condition of perishability and partial backlogging. Manag Sci. 1996; 42(8):1093-104.

16. Chang HJ, Dye CY. An EOQ model for deteriorating items with time varying demand and partial backlogging. J Oper Res Soc. 1999; 50(11):1176-82.

17. Singh TJ, Singh SR, Dutt R. An EOQ model for perishable items with power demand and partial backlogging. Int J Prod Econ. 2009; 15(1): 65-72.

18. Arya RK, Singh SR, Shakya SK. An order level inventory model for perishable items with stock dependent demand and partial backlogging. Int J Comput Appl Math. 2009; 4(1):19-28.

19. Singh SR, Vishnoi M. Supply chain inventory model with price-dependent consumption rate with ameliorating and deteriorating items and two levels of storage. International Journal of Procurement Management. 2013; 6(2):129-51.

20. Singh SR, Kumar T, Gupta CB. A Soft Computing based Inventory model with deterioration and price dependent demand. Int J Comput Appl Tech. 2011; 36(4):10-17. 